\title{
A comparison of postoperative emergence agitation between sevoflurane and thiopental anesthesia induction in pediatric patients
}

\author{
Ji-Seon Son, Eunjoo Jang, Min Wook Oh, Ji-Hye Lee, Young Jin Han, and \\ Seonghoon Ko \\ Department of Anesthesiology and Pain Medicine, Chonbuk National University Medical School and Hospital, \\ Jeonju, Korea
}

\begin{abstract}
Background: This study was performed to compare the incidence of emergence agitation (EA) between inhalation and intravenous anesthesia induction in children after sevoflurane anesthesia.

Methods: In this prospective and double-blind study, 100 children aged 3 to 7 years were enrolled. Subjects were randomly assigned to the sevoflurane (Group S) or thiopental (Group T) anesthesia induction groups. Anesthesia was induced using $8 \%$ sevoflurane and 4-6 mg/kg thiopental in Groups $\mathrm{S}$ and T, respectively. Anesthesia was maintained with nitrous oxide and sevoflurane. The children were evaluated at 5 and $20 \mathrm{~min}$ after arrival in the postanesthesia care unit (PACU) with a four-point agitation scale and the Pediatric Anesthesia Emergence Delirium scale. The incidence of EA and administration of the rescue agent were recorded.

Results: The incidence of EA was significantly lower in Group T compared to Group S at 5 min after PACU arrival (3/49 patients, $6 \%$ vs. $12 / 47$ patients, $26 \%, \mathrm{P}=0.019)$. However, there was no difference between the two groups at $20 \mathrm{~min}$ after PACU arrival (23/49 vs. $19 / 47$ patients in Group T vs. Group S, P = 0.425). The overall incidence of EA was $60 \%(28 / 47$ patients) in Group $S$ and $41 \%(20 / 49$ patients) in Group T ( $\mathrm{P}=0.102)$. The number of children who received propofol as a rescue agent was significantly lower in Group T (Group S: 14/47 vs. Group T: 5/49, P = 0.031).

Conclusions: Intravenous anesthesia induction with thiopental reduced the incidence of EA in the early PACU period compared to inhalation induction with sevoflurane in 3- to 7-year-old children undergoing sevoflurane anesthesia.
\end{abstract}

Key Words: Agitation, Pediatrics, Sevoflurane, Thiopental.

Corresponding author: Seonghoon Ko, M.D., Ph.D.

Department of Anesthesiology and Pain Medicine, Chonbuk National University Medical School and Hospital, 20, Gunji-ro, Deokjin-gu, Jeonju 561-712, Korea

Tel: 82-63-250-1979, Fax: 82-63-250-1240

E-mail: shko@jbnu.ac.kr

Received: August 1, 2014.

Revised: 1st, December 19, 2014; 2nd, December 29, 2014;

3rd, January 30, 2015.

Accepted: February 5, 2015.

Korean J Anesthesiol 2015 August 68(4): 373-378

http://dx.doi.org/10.4097/kjae.2015.68.4.373

\section{Introduction}

Pediatric emergence agitation (EA) is characterized by behaviors during the recovery period following general anesthesia that include inconsolable crying, thrashing, kicking, disorientation, hallucinations, and cognitive and memory impairment [1]. These behaviors can result in numerous potential complications such as falling from the bed, increased postsurgical bleeding, inadvertent removal of drains and catheters, and contamination of the surgical wound $[2,3]$. Although the pathophysiology of EA has not yet been clarified, many previous studies have confirmed

(c) This is an open-access article distributed under the terms of the Creative Commons Attribution Non-Commercial License (http://creativecommons.org/ licenses/by-nc/4.0/), which permits unrestricted non-commercial use, distribution, and reproduction in any medium, provided the original work is properly cited. 
that the incidence of EA in pediatric patients is increased after sevoflurane anesthesia [4-6].

Although inhalation induction by mask with sevoflurane is commonly used in pediatric anesthesia, intravenous induction with intravenous anesthetic is utilized when an intravenous catheter is present or when rapid sequence induction is necessary. Hypnotics used for intravenous anesthesia induction, such as propofol and ketamine, have been reported to reduce the incidence of EA in children [7-11]. There have also been some studies that have compared the incidence of EA between intravenous induction anesthetics $[7,8]$. Although thiopental is a commonly used intravenous induction anesthetic in pediatric anesthesia, there had been no previous controlled trial performed to investigate the effects of thiopental on EA compared to inhalation induction in children. The authors hypothesized that the incidence of EA is lower with the use of thiopental as an induction agent compared to inhalation induction with sevoflurane. The current study was performed to compare the incidence of EA between thiopental and sevoflurane as anesthesia induction agents in children aged 3 to 7 years following sevoflurane anesthesia.

\section{Materials and Methods}

This prospective, double-blind study was approved by the Institutional Review Board of the authors' institute and written informed consent was obtained from the parents of all participants. One hundred children aged 3 to 7 years, American Society of Anesthesiologists physical status I or II, undergoing elective minor superficial surgical procedures were enrolled. Children undergoing emergency or ophthalmic surgery and those with previous anesthetic experience, developmental delays, mental retardation, anxiety disorders, or chronic illnesses were excluded from the study. Subjects were randomly assigned to either the sevoflurane induction group (Group S) or the thiopental induction group (Group T) by a computer-generated random number.

Patients were admitted to the hospital the evening before surgery and were visited by the research anesthesiologist to conduct preoperative interviews. All surgeries were performed in morning sessions. A $24 \mathrm{G}$ catheter was inserted into a peripheral vein the night before surgery or the morning of surgery following application of EMLA (eutectic mixture of local anesthetics) cream in Group T. A balanced salt solution was administered according to standard fluid administration guidelines. No children received premedication in either group. All children were accompanied by their parent in the operating room and the parent was permitted to be present during induction of anesthesia. Children's preoperative anxiety was assessed with the modified Yale Preoperative Anxiety Scale (mYPAS) [12]. After monitor placement with electrocardiography (ECG) and pulse oximetry, the anxiety score using the mYPAS was determined just before anesthesia induction. The mYPAS scores were measured by a single anesthesiologist to exclude inter-rater bias.

In Group S, inhalation anesthesia induction was performed using $8 \%$ sevoflurane and $60 \%$ nitrous oxide $\left(\mathrm{N}_{2} \mathrm{O}\right)$ in oxygen with a fresh gas flow of $8 \mathrm{~L} / \mathrm{min}$. After loss of consciousness, a noninvasive blood pressure cuff was applied and an intravenous line was established. In Group T, the patients were administered 4-6 mg/kg thiopental to induce anesthesia. All patients received $0.6 \mathrm{mg} / \mathrm{kg}$ rocuronium for facilitation of tracheal intubation. Anesthesia was maintained with $50 \% \mathrm{~N}_{2} \mathrm{O}$ in oxygen and sevoflurane and the sevoflurane concentration was controlled at $1.5-3.0 \%$ to maintain the appropriate blood pressure and heart rate. End tidal carbon dioxide $\left(\mathrm{CO}_{2}\right)$ partial pressure was maintained at $30-35 \mathrm{mmHg}$. Additional muscle relaxant was not administered. At skin closure, all patients received $0.25 \%$ ropivacaine infiltration into the incision site and $1 \mathrm{mg} / \mathrm{kg} \mathrm{ke-}$ torolac intravenously for postoperative pain management. The patients also received $0.2 \mathrm{mg} / \mathrm{kg}$ pyridostigmine and $0.01 \mathrm{mg} /$ $\mathrm{kg}$ glycopyrrolate for reversal of the neuromuscular blockade after discontinuation of sevoflurane and $\mathrm{N}_{2} \mathrm{O}$. When the patients were breathing spontaneously with tidal volume $>8 \mathrm{ml} / \mathrm{kg}$, the tracheal tube was removed and the children were transferred to the postanesthesia care unit (PACU). The time from sevoflurane discontinuation to tracheal extubation was recorded.

In the PACU, ECG, respiratory rate, and peripheral oxygen saturation $\left(\mathrm{SpO}_{2}\right)$ were recorded every $5 \mathrm{~min}$, but blood pressure was not measured to avoid physical stimulation. Patients were joined by their parents in the PACU. A data-collecting anesthesiologist who did not perform the anesthesia in the operating room, and was therefore blinded to the anesthesia induction techniques, evaluated children's recovery behaviors in the PACU. The primary endpoint of the study was the incidence of EA in the PACU. The children were evaluated at $5 \mathrm{~min}$ by a four-point agitation scale (FPAS) $[13,14]$ and at $20 \mathrm{~min}$ by the Pediatric Anesthesia Emergence Delirium scale (PAEDS) [1]. The FPAS is based on the following scale: 1 = quiet and calm child; 2 = crying but consolable child; 3 = moderately agitated child: screaming and crying; 4 = aggressive child: must be physically restrained to avoid harm. The children were also evaluated for EA if they showed behavioral changes at any other time in the PACU. EA was defined as an FPAS score $\geq 3$ or a PAED score $\geq 10$. The PAEDS was utilized only after more than 5 min after arrival in the PACU because use of the PAEDS in children who are asleep is inappropriate. The overall incidence of EA was evaluated with the FPAS during the first 5 min in the PACU and with the PAEDS for the next 5-60 min following PACU arrival. If agitation persisted for more than $5 \mathrm{~min}$, children were treated with 1 $\mathrm{mg} / \mathrm{kg}$ propofol at the discretion of the attending anesthesiologist. The number of rescue agent administrations was recorded. 


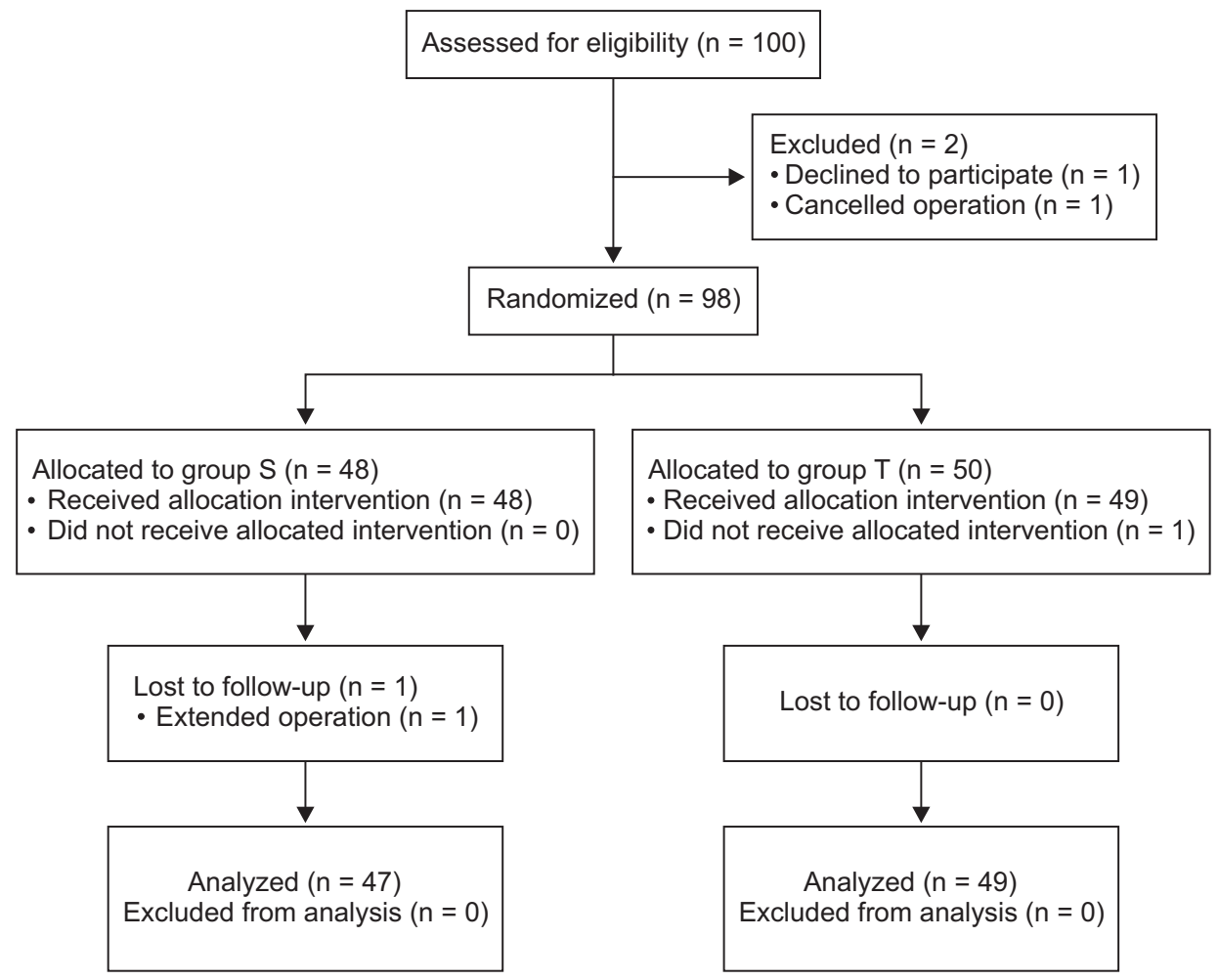

Fig. 1. Subject flow diagram. Group S: sevoflurane inhalation anesthesia induction group, Group T: intravenous thiopental anesthesia induction group.

\section{Sample size determination and statistical analysis}

The incidence of EA in children undergoing anesthesia induction with sevoflurane was assumed to be $65 \%$ to determine the appropriate sample size. According to a sample size calculation determined by proportions sample size, 42 children per group were required $(\alpha=0.05, \beta=0.2)$ to detect a $50 \%$ reduction in Group T. To allow for attrition, the sample size was enlarged to 100 .

Data were analyzed using SigmaPlot 12.0 (Systat Software Inc., San Jose, CA, USA), and are presented as a number (n), percentage (\%), mean \pm standard deviation (SD), or median \pm interquartile range (IQR) as appropriate. The Student's $t$-test was used to compare the mean value of quantitative data between the two groups. Nonparametric data such as the PAEDS and FPAS scores were compared between the groups with the Mann-Whitney $U$ test. Categorical data such as the incidence of EA and the number of patients requiring rescue drugs were compared by the chi-squared test and expressed as a number or percentage. A P value of $<0.05$ was considered statistically significant in all tests.

\section{Results}

Ninty-six children completed the study and the subject flow diagram is show in Fig. 1. The characteristics of the patients are
Table 1. Patient Characteristics

\begin{tabular}{lccc}
\hline & $\begin{array}{c}\text { Group S } \\
(\mathrm{n}=47)\end{array}$ & $\begin{array}{c}\text { Group T } \\
(\mathrm{n}=49)\end{array}$ & P values \\
\hline Age $(\mathrm{yr})$ & $4.3 \pm 0.8$ & $4.4 \pm 1.4$ & 0.520 \\
Height $(\mathrm{cm})$ & $104.9 \pm 9.2$ & $108.1 \pm 11.7$ & 0.139 \\
Weight $(\mathrm{kg})$ & $18.9 \pm 4.7$ & $18.8 \pm 4.5$ & 0.920 \\
Gender (F/M) & $19 / 28$ & $18 / 31$ & 0.872 \\
Fasting time (h) & $11.4 \pm 1.9$ & $11.7 \pm 1.9$ & 0.469 \\
Anesthesia time (min) & $54.0 \pm 14.9$ & $57.6 \pm 14.8$ & 0.241 \\
Surgery & & & 0.988 \\
$\quad$ Herniorrhaphy & 32 & 33 & \\
$\quad$ Hand surgery & 9 & 8 & \\
$\quad$ Other & 6 & 8 & \\
\hline
\end{tabular}

Data are expressed as mean \pm SD except number of patients and types of surgery. Group S: sevoflurane inhalation anesthesia induction group. Group T: intravenous thiopental anesthesia induction group.

comparable in both groups (Table 1). The median preoperative anxiety scores using the mYPAS in the operating room were not different between the two groups (Group S: 31.7 [23.3-40.0] vs. Group T: 31.7 [25.0-46.7], $\mathrm{P}=0.211$ ). The time from the end of sevoflurane administration to extubation was also similar in the two groups (Group S: $6.8 \pm 2.8$ min vs. Group T: $7.5 \pm 3.2 \mathrm{~min}$, $\mathrm{P}=0.258)$.

The incidence of EA (Group T: 3/49 patients, 6\% vs. Group S: 12/47 patients, $26 \%, \mathrm{P}=0.019$ ) and the FPAS scores (Group T: 1.0 [1.0-2.0] vs. Group S: 2.0 [1.0-3.0], P = 0.024) were significantly 
lower in Group T compared to Group S at 5 min after PACU arrival. However, there were no differences in the incidence of EA (Group S: 23/47 patients vs. Group T: 19/49 patients, $\mathrm{P}=0.425$ ) or PAEDS (Group S: 8.0 [0.0-12.0] vs. Group T: 7.0 [4.0-10.5], P $=0.580$ ) between the two groups at $20 \mathrm{~min}$ (Fig. 2). The overall incidence of EA was 60\% (28/47 patients) in Group S and 41\% (20/49 patients) in Group $\mathrm{T}(\mathrm{P}=0.102)$ (Fig. 2). The distribution of EA scores was significantly different between the groups at 5 min after PACU arrival $(\mathrm{P}=0.045)$, but did not differ at $20 \mathrm{~min}$ after PACU arrival $(\mathrm{P}=0.848)$ (Table 2$)$. The number of children who received propofol as a rescue drug for treatment of EA was significantly lower in Group T (Group S: 14/47 patients vs. Group T: 5/49 patients, $\mathrm{P}=0.031$ ). No patient showed EA after $30 \mathrm{~min}$ in the PACU. No adverse events including respiratory complications such as desaturation associated with EA or the rescue drug were recorded.

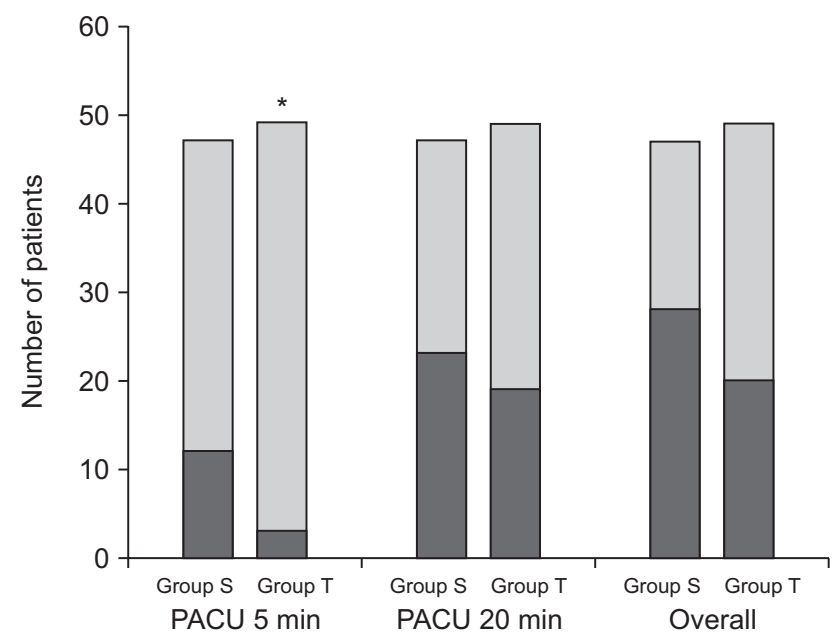

Fig. 2. There was a significant difference in the incidence of emergence agitation (EA) between the two groups at $5 \mathrm{~min}$ after arrival in the postanesthesia care unit (PACU) ( 3 vs. 12 patients, $* \mathrm{P}=0.019)$. However, there were no differences at $20 \mathrm{~min}(23$ vs. 19 patients, $\mathrm{P}=0.425)$ or in the overall incidence $(\mathrm{P}=0.102)$. The black stack presents the number of patients who showed EA. Group S: sevoflurane inhalation anesthesia induction group, Group T: intravenous thiopental anesthesia induction group.

\section{Discussion}

The objective of the current study was to compare the incidence of EA between inhalation and intravenous anesthesia induction. The results of this study demonstrate that intravenous anesthesia induction with thiopental decreased the incidence of EA and the use of rescue medication compared to inhalation induction in the early PACU period in 3- to 7-year-old children undergoing sevoflurane anesthesia.

The terms emergence delirium and emergence excitement are often used as alternatives to EA. Although EA is a complex phenomenon and its pathophysiology is as yet unclarified, the age of the patient and the anesthetic agents used might be major contributing factors. In younger children, the higher incidence of EA may be related to psychological immaturity and lower ability to cope in a strange environment upon awakening from general anesthesia [14,15]. A number of studies have demonstrated that higher incidence of EA is associated with a volatile anesthetic which has lower blood solubility, such as sevoflurane and desflurane $[5,6,13,16,17]$. Rapid awakening from anesthesia combined with psychological immaturity has been postulated as one explanation for the pathophysiology of EA [14]. The incidence of EA varies widely between 25 and $80 \%$ following sevoflurane or desflurane anesthesia in preschool children $[2,18,19]$. In the current study, the overall incidence was 60 and $41 \%$ in Groups S and T, respectively. These rates were comparable with the findings of previous studies, although more than $90 \%$ of the children in the present study were $\leq 5$ years of age.

The differences in the incidence of EA in the previous studies may be due to differences in measurement timing and scoring systems for EA in the PACU [19]. In the current study, children were evaluated at 5 and 20 min after arrival in the PACU and no child displayed EA after $30 \mathrm{~min}$, a finding which supports previous studies suggesting that EA occurs during the first $30 \mathrm{~min}$ of recovery from anesthesia [19-21]. The present study used the PAEDS and the FPAS to measure agitation scores. Although the PAEDS is the best discriminator of EA [22,23], it is inappropriate in sleeping children [24]. The FPAS is a more appropriate scoring

Table 2. The Distribution of Patients according to Emergence Agitation Scales

\begin{tabular}{|c|c|c|c|c|}
\hline & & Group S $(n=47)$ & Group T $(\mathrm{n}=49)$ & $\mathrm{P}$ values \\
\hline \multirow[t]{4}{*}{ FPAS (5 min after PACU arrival) } & 1 & 21 & 33 & \multirow{4}{*}{0.045} \\
\hline & 2 & 14 & 13 & \\
\hline & 3 & 8 & 2 & \\
\hline & 4 & 4 & 1 & \\
\hline \multirow[t]{4}{*}{ PAEDS (20 min after PACU arrival) } & $0-5$ & 15 & 19 & \multirow{4}{*}{0.848} \\
\hline & $6-9$ & 14 & 15 & \\
\hline & $10-15$ & 15 & 13 & \\
\hline & $>15$ & 3 & 2 & \\
\hline
\end{tabular}

FPAS: four-point agitation scale, PAEDS: Pediatric Anesthesia Emergence Delirium scale, PACU: postanesthesia care unit, Group S: sevoflurane inhalation anesthesia induction group, Group T: intravenous thiopental anesthesia induction group. 
system than the PAEDS before the child has awakened. Therefore, the authors used the FPAS within 5 min and the PAEDS after $5 \mathrm{~min}$ in the PACU to evaluate the incidence and severity of EA.

Although propofol is the most commonly employed agent for intravenous induction of anesthesia, it causes burning pain at the site of injection. On the other hand, because thiopental does not cause pain with injection, it is used commonly as an intravenous induction agent in pediatric patients. However, the prolonged elimination half-life of thiopental can result in prolonged lethargy and after effects that last for hours after emergence from anesthesia [18]. The long-lasting subhypnotic effect of thiopental may have contributed to the lower incidence of EA in Group T in the early PACU period although there was no significant difference in the time from the end of sevoflurane administration to extubation between the two groups. The authors expected that the lower incidence of EA would be maintained throughout the patients' stay in the PACU, but the effect was observed only in the early PACU period.

EA has been shown to be associated with postoperative pain. The efficacy of analgesics such as fentanyl in the prevention or treatment of EA could be explained by their effects on postoperative pain. Although postoperative pain seems to be related to EA, it can also occur in pain-free children [16]. A meta-analysis demonstrated that analgesic effects had no influence on the efficacy of drugs administered for EA [25]. In addition, Cole et al. [26] reported that the type of operation or method of pain management did not alter the risk for EA. However, categories of EA scoring systems overlap with pain scales for children such as the Face, Legs, Activity, Cry, Consolability scale and the Children's and Infants' Postoperative Pain Scale. Therefore, it can be difficult to distinguish EA from postoperative pain in preschool children.
High preoperative anxiety has been associated with the development of negative behaviors [27,28]. Kain et al. [29] proposed that preoperative anxiety is one of the contributing factors to EA. A number of preoperative preparation programs are used for pediatric surgical patients to alleviate preoperative anxiety and could prevent postoperative negative behavioral changes. Because most children have fear of needles, the authors thought that the intravenous catheterization in Group $\mathrm{T}$ could be a source of increased preoperative anxiety. However, there were no differences in the mYPAS scores between the two groups in the current study. Although the parent was present during the anesthesia induction, most patients cried or refused the mask, which may be a source of increased preoperative anxiety coinstantaneously. However, the anxiety associated with the mask was not evaluated in this study.

There are two limitations in the current study. First, the sedation score was not measured in the PACU. Although the plasma concentration of thiopental is redistributed and the hypnotic effect is completed rapidly, it may be affect the sedation and EA scores in the early PACU period. If the authors evaluated the sedation score thoroughly, the scores might be found to differ between the two groups. Second, the patient's pain state was not evaluated. Many categories of EA scoring systems are overlapped with pain scales for children. Although the patients received ropivacaine infiltration and ketorolac to maintain a painless state in the PACU, the authors could not eliminate the effect of pain on EA. The authors, however, thought that the effect of pain on EA may not be different between the two groups.

In conclusion, intravenous anesthesia induction with thiopental reduced the incidence of EA in the early PACU period compared to inhalation induction with sevoflurane in 3- to 7-year-old children undergoing sevoflurane anesthesia.

\section{References}

1. Sikich N, Lerman J. Development and psychometric evaluation of the pediatric anesthesia emergence delirium scale. Anesthesiology 2004; 100: $1138-45$

2. Voepel-Lewis T, Malviya S, Tait AR. A prospective cohort study of emergence agitation in the pediatric postanesthesia care unit. Anesth Analg 2003; 96: 1625-30.

3. Uezono S, Goto T, Terui K, Ichinose F, Ishguro Y, Nakata Y, et al. Emergence agitation after sevoflurane versus propofol in pediatric patients. Anesth Analg 2000; 91: 563-6.

4. Welborn LG, Hannallah RS, Norden JM, Ruttimann UE, Callan CM. Comparison of emergence and recovery characteristics of sevoflurane, desflurane, and halothane in pediatric ambulatory patients. Anesth Analg 1996; 83: 917-20.

5. Cravero J, Surgenor S, Whalen K. Emergence agitation in paediatric patients after sevoflurane anaesthesia and no surgery: a comparison with halothane. Paediatr Anaesth 2000; 10: 419-24.

6. Picard V, Dumont L, Pellegrini M. Quality of recovery in children: sevoflurane versus propofol. Acta Anaesthesiol Scand 2000; 44: 307-10.

7. Hwang SM, Kim KS, Lim SY. Comparison of emergence agitation from sevoflurane anesthesia in propofol or thiopental sodium induction. Korean J Anesthesiol 2004; 46: 647-51.

8. Kim HS, Lee SK, Kang HS, Kim YM, Choi H, Moon HS. Comparison of thiopental sodium and ketamine induction on emergence agitation 
after desflurane anesthesia in children undergoing a tonsillectomy. Korean J Anesthesiol 2007; 53: 356-60.

9. Abu-Shahwan I. Effect of propofol on emergence behavior in children after sevoflurane general anesthesia. Paediatr Anaesth 2008; 18 : 55-9.

10. Guler G, Akin A, Tosun Z, Ors S, Esmaoglu A, Boyaci A. Single-dose dexmedetomidine reduces agitation and provides smooth extubation after pediatric adenotonsillectomy. Paediatr Anaesth 2005; 15: 762-6.

11. Khattab AM, El-Seify ZA. Sevoflurane-emergence agitation: Effect of supplementary low-dose oral ketamine premedication in preschool children undergoing dental surgery. Saudi J Anaesth 2009; 3: 61-6.

12. Kain ZN, Mayes LC, Cicchetti DV, Bagnall AL, Finley JD, Hofstadter MB. The Yale Preoperative Anxiety Scale: how does it compare with a "gold standard"? Anesth Analg 1997; 85: 783-8.

13. Finkel JC, Cohen IT, Hannallah RS, Patel KM, Kim MS, Hummer KA, et al. The effect of intranasal fentanyl on the emergence characteristics after sevoflurane anesthesia in children undergoing surgery for bilateral myringotomy tube placement. Anesth Analg 2001; 92: 1164-8.

14. Aono J, Ueda W, Mamiya K, Takimoto E, Manabe M. Greater incidence of delirium during recovery from sevoflurane anesthesia in preschool boys. Anesthesiology 1997; 87: 1298-300.

15. Wells LT, Rasch DK. Emergence "delirium" after sevoflurane anesthesia: a paranoid delusion? Anesth Analg 1999; 88: 1308-10.

16. Cravero JP, Beach M, Thyr B, Whalen K. The effect of small dose fentanyl on the emergence characteristics of pediatric patients after sevoflurane anesthesia without surgery. Anesth Analg 2003; 97: 364-7.

17. Lapin SL, Auden SM, Goldsmith LJ, Reynolds AM. Effects of sevoflurane anaesthesia on recovery in children: a comparison with halothane. Paediatr Anaesth 1999; 9: 299-304.

18. Polaner DM. Management of general Anesthesia. In: Pediatric Anesthesia. Edited by Holzman RS, Mancuso TJ, Polaner DM: Philadelphia, Lippincott Williams \& Wilkins. 2008, pp 100-3.

19. Vlajkovic GP, Sindjelic RP. Emergence delirium in children: many questions, few answers. Anesth Analg 2007; 104: 84-91.

20. Malarbi S, Stargatt R, Howard K, Davidson A. Characterizing the behavior of children emerging with delirium from general anesthesia. Paediatr Anaesth 2011; 21: 942-50.

21. Hudek K. Emergence delirium: a nursing perspective. AORN J 2009; 89: 509-16.

22. Aouad MT, Yazbeck-Karam VG, Nasr VG, El-Khatib MF, Kanazi GE, Bleik JH. A single dose of propofol at the end of surgery for the prevention of emergence agitation in children undergoing strabismus surgery during sevoflurane anesthesia. Anesthesiology 2007; 107: 733-8.

23. Bong CL, Ng AS. Evaluation of emergence delirium in Asian children using the Pediatric Anesthesia Emergence Delirium Scale. Paediatr Anaesth 2009; 19: 593-600.

24. Park JH, Lim BG, Lee IO. The effect of sedation on the pediatric anesthesia emergence delirium scale. Paediatr Anaesth 2014; 24: 1014-5.

25. Dahmani S, Stany I, Brasher C, Lejeune C, Bruneau B, Wood C, et al. Pharmacological prevention of sevoflurane- and desflurane-related emergence agitation in children: a meta-analysis of published studies. Br J Anaesth 2010; 104: 216-23.

26. Cole JW, Murray DJ, McAllister JD, Hirshberg GE. Emergence behaviour in children: defining the incidence of excitement and agitation following anaesthesia. Paediatr Anaesth 2002; 12: 442-7.

27. Lumley MA, Melamed BG, Abeles LA. Predicting children’s presurgical anxiety and subsequent behavior changes. J Pediatr Psychol 1993; 18: 481-97.

28. McCann ME, Kain ZN. The management of preoperative anxiety in children: an update. Anesth Analg 2001; 93: 98-105.

29. Kain ZN, Caldwell-Andrews AA, Maranets I, McClain B, Gaal D, Mayes LC, et al. Preoperative anxiety and emergence delirium and postoperative maladaptive behaviors. Anesth Analg 2004; 99: 1648-54. 